inhibition of the action of urokinase was produced by unheated epidermis but not by the heated epidermis.

Serial dilutions of blister fluid and of citrated plasma were similarly studied and were found to have inhibitory action. 'To rule out contamination of epidermis by blister fluid, equal weights of epidermis, blister fluid and plasma were suspended in equal volumes of veronal buffer. Serial dilutions of these demonstrated greatest inhibition by the epidermis.

These findings indicate that human epidermis can inhibit fibrinolysis. The experiments do not define the nature of the inhibitory factor: that is, whether enzymatic and, if so, its site of action. Further experiments are needed to demonstrate inhibition of the lysis of human fibrin containing plasminogin. The inhibition produced by blister fluid is most likely derived from inhibitors in plasma, but some contribution by epidermis is possible.

This project was supported by the Herbert E. Dunhill Trust; R. H. T. was the recipient of a Gillette research fellowship.

Institute of Dermatology,

Robert H. TURner

AMAL. K. KURBAN

Terence J. Ryan

St John's Hospital for Diseases of the Skin,

London WC2.

Received May 22, 1969.

${ }^{1}$ Pugatch, E. M. J., and Poole, J. C. F., Nature, 221, 269 (1969).

2 Todk, A. S., Vature, 181, $495(1958)$.

${ }^{3}$ Kiistala, C., J. Invest. Derm., 50, 129 (1968).

\section{Tetanus Toxin, a Neuromuscular Blocking Agent}

In some cases of infectious tetanus a neuromuscular block with tetraplegia may follow the stato of muscle spasms. We suggested ${ }^{1}$ that this effect was caused by the tetanus toxin itself rather than to an after-effect of muscle relaxants or other drugs. 'This hypothesis, however, had to be verified by experiments with animals.

Local tetanus in rats appeared to be the best way to study the effect of tetanus toxin on the neuromuscular junction. The toxin was injected in various amounts and dilutions into one hindleg and the animals were tested at various intervals after the injection. Under urethane anaesthesia the peroneal nerve was cut in the thigh, and the distal stump stimulated by single and repetitive clectric shocks. The tension of the anterior tibial muscle was registered by a strain-gauge attached to the tendon. The "healthy" opposite hindleg always served as a control. In addition, various nouromuscular blocking agents such as magnesium sulphate, 'Alloferin' and succinylcholine were tosted in identical experimental conditions.

The results can be summarized as follows: In severe local tetanus neuromuscular transmission may be abolished, for example, the rnuscle becomes unresponsive to indirect but remains fully responsive to direct electrical stimulation. A small response to a single supramaximal stimulus and a further decrement of amplitude on repetitive stimulation are observed in other instances. With tetanic stimulation the tension becomes almost equal to that of the healthy side. Post-tetanic potentiation is marked (up to 300 per cent) whereas post-tetanic depression is lacking or mild. Edrophonium chloride ('Tensilon) and 'Neostigmin' improved the transmission only to a moderate extent. The neuromuscular block produced by tetanus toxin is similar to that observed in man and to that in rat after injection of magnesium sulphate.

Our experiments in rats thus confirm that tetanus toxin, besides its central action causing muscle spasms, also had a peripheral effect interfering with neuromuscular transmission. It seems likely that tetanus toxin inhibits the release of acetylcholine from the nerve terminals, but a post-synaptic action cannot be ruled out without further investigations. In man, the peripheral action of the toxin may be responsible for the so-called recruiting response on voluntary eontraction, for circumscript muscle weakness in local tetanus and for tetraplegia observed in a small percentage of patients with severo tetanus. Damage to the muscle fibres causing a reversible myopathy is another feature that may be responsible for the slow recovery of muscle strength.

H. F. Kaeser

A. SANER

Neurological Clinic,

University of Basle,

Switzerland.

Received June 4, 1969.

${ }^{1}$ Kaeser, H. \&., Yüller, H. R., an:I Friedrich, B., Ewrop. Netrolog., 1, 17 (1968).

\section{Additional Site for the Action of Cholera Toxin}

AsIatre cholera is a disease of the gastrointestinal tract and its cardinal symptom is "rice-water" diarrhoea. The primary site of pathology is the intestinal canal where electrolytes and fluid are poured in the lumen and lost to the body. Primary systemic effects, if any, play littlo part in the pathogenesis of cholera. The gastrointestinal tract has naturally been the target of study for the action of cholera toxins ${ }^{1-3}$. Cireenough ${ }^{4}$, however, provided strong evidence for the involvement of the duodenum by demonstrating that the aspiration of the duodenal contents in cholera patients reduced the diarrhoeal volume. Finkelstein et al. ${ }^{5}$ found in infant rabbits ${ }^{6}$ that the caccum is unresponsive to cholera toxin, while Leitch and Burrows ${ }^{7}$ found the colon of rabbit unresponsive to cholera toxin. Surprisingly enough, no studies have been carried out on the stomach, probably because no vibrio can survive in the acidic conditions of the stomach.

We have found that the stomach is responsive to cholera toxin and here we report our preliminary finding:s and their significance. Fight to twelve day old infant rabbits of either sex, bred at the Haffkine Institute, were used in the present experiments. The animals were given water freely for $48 \mathrm{~h}$ before being anaesthetized with ether, and their abdomens were opened and loops of the stomach formed by tying ligatures at both the pyloric and cardiac ends. One more ligature was passed round the duodenum near the pyloric end and the continuity between the stomach and duodenum was severed by cutting in between the ligatures. In the loop $15 \mathrm{mg}$ of syncase toxin ${ }^{6}(0.5 \mathrm{ml}$.) was injected while the control group received $0.5 \mathrm{ml}$. of water. The abdomen was stitched and animals observed for $24 \mathrm{~h}$. At the end of this period, the animals were killed and the loops examined. Fluid from the loop was withdrawn, measured and analysed for sodium, potassium and chloride. The metal ions were determined by standard flame photometry ${ }^{8}$. while the chlorine was estimated by tho procedure of Whitehorn ${ }^{9} . \quad p \mathrm{H}$ determinations were made using $\mathrm{E}$. Merck's narrow range $p \mathrm{H}$ papers.

The control loops were dry, while those recoiving toxin were full of fluid. The $p \mathrm{H}$ was highly acid $(1.5-1 \cdot 8)$, and large amounts of sodium and chlorides were present.

It is very clear that the stomach responds vigorously to the toxin and pours out into the lumen its normal secretions, hydrochloric acid and sodium ions. The volume of accumulated fluid amounted to about 10 per cent of the body weight of the animal and this is very significant in view of the observation that when the loss of fluid reaches to the level of $10-15$ per cent of the body weight, the animals do not survive ${ }^{10}$.

This raises the question of the possibility of the presence of cholera toxin in the stomach, for the cultivation of 